\title{
Exaggerated Atrial Arachidonate Metabolism in Rabbit Left Ventricular Myocardial Infarction
}

\author{
Alex S. Evers, Carol G. Dunkel, Jeffrey E. Saffitz," and Philip Needleman* \\ Departments of Anesthesiology, Pharmacology ${ }^{\ddagger}$ and Pathology, ${ }^{*}$ Washington University School of Medicine, St. Louis, Missouri 63110
}

\begin{abstract}
Isolated perfused rabbit hearts that have previously been subjected to in vivo left ventricular myocardial infarction respond to $\boldsymbol{N}$-formylmethionyl-leucyl-phenylalanine (fMLP) or bradykinin (BK) administration with the synthesis of large quantities of eicosanoids. To anatomically localize these synthetic responses we studied the effects of fMLP and BK on eicosanoid synthesis in isolated atria and isolated perfused ventricles from normal and infarcted ( $4 \mathrm{~d}$ in vivo) rabbit hearts. These studies revealed that enhanced agonist-stimulated eicosanoid synthesis occurs largely in the right atria of infarcted hearts, a site distant from the zone of injury. Studies of exogenous arachidonate metabolism in microsomes prepared from various regions of the heart showed that while prostaglandin synthetic capacity is preferentially localized to the right atrium, right atria from normal and infarcted hearts have similar thromboxane and $\mathrm{PGE}_{2}$ synthetic capacity. These results demonstrate that enhanced agonist-stimulated eicosanoid synthesis following rabbit left ventricular myocardial infarction occurs largely in the right atrium, and that this effect is independent of the activity of prostaglandin synthetic enzymes.
\end{abstract}

\section{Introduction}

Several investigations have demonstrated that infarcted canine myocardium has increased enzymatic capacity for the synthesis of thromboxane $A_{2}$, prostaglandin $E_{2}$, prostacyclin (1) and 12hydroxyeicosatetraenoic acid $(2,3)$. In these studies enhanced eicosanoid synthetic capacity was temporally correlated with the appearance of polymorphonuclear leukocytes and macrophages in the infarcted tissue (1-3). The functional significance of enhanced cardiac eicosanoid synthesis in myocardial infarction has been suggested by several lines of investigation. The combined cyclooxygenase-lipoxygenase inhibitor $\mathrm{BW}-755 \mathrm{C}$ has been shown to both decrease infarct size and reduce the incidence of ventricular dysrythmias in experimental canine myocardial infarction $(2,4,5)$. The lack of a myocardial sparing effect for pure cyclooxygenase inhibitors such as aspirin (6), indomethacin (7), and naproxen (8), suggests the involvement of lipoxygenase metabolites of arachidonic acid in myocardial infarct extension.

In a previous study we described a rabbit model of in vivo left ventricular myocardial infarction followed by ex vivo cardiac perfusion (9). Using this model we demonstrated that $N$-for-

\footnotetext{
Address reprint requests to Dr. Needleman.

Received for publication 19 March 1986 and in revised form 30 July 1986.
}

J. Clin. Invest.

(C) The American Society for Clinical Investigation, Inc. 0021-9738/87/01/0155/08 \$1.00

Volume 79, January 1987, 155-162 mylmethionyl-leucyl-phenylalanine (fMLP) ${ }^{1}$ and bradykinin (BK) stimulate the synthesis of large amounts of thromboxane, prostaglandin $\mathrm{E}_{2}\left(\mathrm{PGE}_{2}\right)$, and prostacyclin in infarcted, but not control rabbit hearts. While fMLP also stimulated the synthesis of leukotrienes $B_{4}, C_{4}$, and $D_{4}$ in the isolated perfused rabbit heart, leukotriene synthesis was quantitatively similar in control and infarcted hearts. It is interesting that fMLP caused a coronary vasoconstriction in infarcted but not control rabbit hearts, that was specifically inhibited by the peptidoleukotriene receptor antagonist FPL-55712 (9). Exaggerated prostanoid synthesis in infarcted rabbit hearts was temporally associated with the influx of inflammatory cells into the infarcted tissue. The association between inflammatory cell invasion and exaggerated cardiac arachidonate metabolism was strengthened by the observation that $30 \mathrm{~d}$ after infarction inflammatory cells had disappeared from the infarct zone, in association with markedly reduced prostanoid synthesis (9).

In the current study we have extended our previous observations by anatomically localizing the sites of cardiac arachidonate metabolizing enzymes, of agonist-stimulated arachidonate metabolism, and of the PMLP-induced coronary vasoconstriction. We now report the unexpected discovery that the enzymatic machinery for cardiac arachidonate metabolism is concentrated in the right cardiac atrium. Furthermore, the enhanced agonist-stimulated arachidonate metabolism and fMLPinduced coronary vasoconstriction observed in infarcted rabbit hearts are also preferentially localized to the right atrium.

\section{Methods}

Materials. $\left[{ }^{3} \mathrm{H}\right] 6-k e t o$ prostaglandin $\mathrm{F}_{1 \alpha}(160 \mathrm{Ci} / \mathrm{mM}),\left[{ }^{3} \mathrm{H}\right]$ leukotriene $\mathrm{C}_{4}(40 \mathrm{Ci} / \mathrm{mM})$ and ${ }^{57} \mathrm{Co}$ and ${ }^{51} \mathrm{Cr}$-labeled microspheres were purchased from New England Nuclear (Boston, MA). Carrier-free [ $\left.{ }^{125} \mathrm{I}\right] \mathrm{NaI}$ (14 mCi/ $\mu \mathrm{g}$ iodine) and $\left[{ }^{14} \mathrm{C}\right]$ arachidonic acid $(60 \mathrm{Ci} / \mathrm{mM})$ were purchased from Amersham Corp. (Arlington Heights, IL). Prostaglandin standards were the gift of Dr. John Pike of Upjohn Co. (Kalamazoo, MI); synthetic leukotriene $\mathrm{C}_{4}\left(\mathrm{LTC}_{4}\right)$ was a gift of Dr. J. Rokach of Merck-Frosst Laboratories (Duval, Quebec, Canada); indomethacin was a gift from Merck \& Co. (Rahway, NJ); and BW-755C was a gift from Burroughs Wellcome (Research Park, NC). LTC $_{4}$ antisera was kindly supplied by Dr. R. Bell (Riker Laboratories, St. Paul, MN), $N$-formylmethionyl-leucyl-phenylalanine and triphenyl tetrazolium chloride were purchased from Sigma Chemical Co. (St. Louis, MO), and BK was purchased from BoehringerMannheim (Indianapolis, IN). Silica gel plates for thin-layer chromatography $(20 \times 20 \mathrm{~cm} ; 0.25 \mathrm{~mm})$ were purchased from Analabs (North Haven, CT) and $3 A 70$ scintillation cocktail for aqueous samples was purchased from Research Products (Mt. Prospect, IL). Other chemicals were reagent or high performance liquid chromatography grade.

Surgical myocardial infarction. Left ventricular myocardial infarction was surgically produced in male New Zealand white rabbits $(2-3 \mathrm{~kg})$ as

1. Abbreviations used in this paper: BK, bradykinin; fMLP, $N$-formylmethionyl-leucyl-phenylalanine; $\mathrm{LTC}_{4}$, leukotriene $\mathrm{C}_{4}$; PG, prostaglandin; $\mathrm{TxB}_{2}$, thromboxane $\mathrm{B}_{2} ; V_{\max }$, maximum velocity. 
previously described (9). The protocol consisted of $60 \mathrm{~min}$ of occlusion of the distal left circumflex coronary artery followed by reperfusion. Following chest closure the animals were allowed to convalesce for $\mathbf{4 d}$.

Heart perfusion. After the convalescence period, the animals were reanesthetized (pentobarbital, $30 \mathrm{mg} / \mathrm{kg}$ i.v.), heparin was administered ( $500 \mathrm{U}$ i.v.), and the hearts were excised and placed in ice-cold KrebsHenseleit buffer. The aorta was rapidly cannulated, and the hearts were placed in a warming jacket and perfused (Langendorff technique) with oxygenated $\left(\mathrm{O}_{2} / \mathrm{CO}_{2}, 95: 5 \%\right)$ Krebs-Henseleit buffer at $37^{\circ} \mathrm{C}$ and $25 \mathrm{ml} /$ $\mathrm{min}$. The mean coronary perfusion pressure was monitored via a sidearm in the perfusion apparatus using a pressure transducer (P23db, Statham, Inc., Oxnard, CA) and a Gould 440 brush recorder (Gould, Inc., Santa Clara, CA).

To prepare isolated perfused ventricles, hearts were cannulated and perfused as described above. The atria were then removed by cutting along the atrioventricular groove with care taken to avoid the left coronary artery. After removal of the cardiac atria, spontaneous rate of contraction decreased from $150-200 / \mathrm{min}$ to $40-50 / \mathrm{min}$. The coronary perfusion flow rate was decreased to $20 \mathrm{ml} / \mathrm{min}$ to maintain coronary perfusion pressure at $50-60 \mathrm{~mm} \mathrm{Hg}$. Minimal leak of the perfusate occurred from the cut atrioventricular groove. In both isolated perfused hearts and isolated perfused ventricles, 5 -min samples ( 125 or $100 \mathrm{ml}$, respectively) of coronary venous effluent were collected before and after intracoronary bolus administration of either fMLP or BK. A 1-ml aliquot of the effluent was saved $\left(-20^{\circ} \mathrm{C}\right)$ for subsequent prostaglandin radioimmunoassays (RIA), and the remainder of the effluent was extracted (as described below) for $\mathrm{LTC}_{4}$ RIA. At the end of each experiment the hearts were perfused with $100 \mathrm{ml}$ of triphenyl tetrazolium chloride to confirm infarction.

To prepare isolated atria, hearts were removed from rabbits as described above. The atria were removed en masse by cutting along the atrioventricular groove. Vascular tissue and fat were trimmed from the atria, ligatures were placed on the tip of the right and left atrial appendages and the atria were suspended under $1 \mathrm{~g}$ of tension in a tissue bath containing $20 \mathrm{ml}$ of continuously oxygenated $\left(\mathrm{O}_{2} / \mathrm{CO}_{2}, 95: 5 \%\right) \mathrm{Krebs}-\mathrm{Hen}$ seleit buffer at $37^{\circ} \mathrm{C}$. These preparations spontaneously beat at a rate of $\sim 150 / \mathrm{min}$. Isolated right and left atria were prepared in an analogous fashion, with the exception that the two atria were separated by cutting through the interatrial septum and placed in separate tissue baths. Right atrial preparations spontaneously contracted at a rate of $\sim 150 / \mathrm{min}$, whereas left atrial preparations showed no spontaneous contractile activity. The buffer in the tissue baths was changed every $10 \mathrm{~min}$ for $\mathbf{3 0}$ min. At this time buffer was collected after either a 5-min control period, or a 5-min exposure to either fMLP or BK. 20-min recovery periods were allowed between agonist stimulations. A 1-ml aliquot of the bath buffer was saved $\left(-20^{\circ} \mathrm{C}\right)$ for prostaglandin RIA and the remainder of the buffer was extracted for $\mathrm{LTC}_{4}$ RIA.

$R I A$. Basal and poststimulation samples were analyzed for 6-keto PGF $_{1 \alpha}$ concentration using a specific RIA as previously described (9). Samples were analyzed for $\mathrm{PGE}_{2}$ concentration by a previously described RIA using ${ }^{125} \mathrm{I}_{-} \mathrm{PGE}_{2}$ as the radioligand (10). Samples were analyzed for thromboxane B2 (TxB2) using a ${ }^{125}$ I radioimmunoassay. ${ }^{125} \mathrm{I}-\mathrm{TxB} 2$ was prepared by the method of Maclouf (11). The assay was performed as follows: either standards or samples were added to $50 \mathrm{mM}$ phosphatebuffered saline containing 0.01 M EDTA, $0.3 \%$ bovine $\gamma$-globulin, $0.005 \%$ Triton $\mathrm{X}-100$, and $0.05 \%$ sodium azide (pH 6.8) to a total volume of $160 \mu \mathrm{l} .20 \mu \mathrm{l}$ of antisera (final dilution 1:40,000) was added, followed by $20 \mu \mathrm{l}$ of ${ }^{125} \mathrm{I}-\mathrm{TxB}_{2}$ containing $10,000 \mathrm{dpm}$. After overnight incubation at $4^{\circ} \mathrm{C}, 16 \%$ polyethylene glycol in $50 \mathrm{mM}$ phosphate-buffered saline was added to the tubes. The precipitated bound tracer was separated by centrifugation at $3,000 \mathrm{rpm}$ for $30 \mathrm{~min}$ and decanting the supernatant. The tubes were counted in an automatic gamma counter (Apex, Micromedic Systems, Inc., Horsham, PA).

Before $\mathrm{LTC}_{4} \mathrm{RIA}$, samples of coronary effluent or atrial bath buffer were acidified to $\mathrm{pH} 6.2$ with $\mathrm{HCl}$ and applied to a preactivated $1-\mathrm{g}$ open bed C-18 octadecylsilyl column (J. T. Baker Chemical Co., Phillipsburg, $\mathrm{NJ})$. The column was washed with water and petroleum ether and products were eluted with $10 \mathrm{ml}$ of $100 \%$ methanol. In selected experiments $\left[{ }^{3} \mathrm{H}\right] \mathrm{LTC}_{4}$ was added to the perfusate to monitor recovery from the octadecylsilyl column. Recoveries varied from 70 to $81 \%$. Aliquots of the methanol (30-300 $\mu \mathrm{l})$ were placed in siliconized glass tubes and dried under $\mathrm{N}_{2}$. LTC 4 RIA was performed in these tubes as previously described (9). The crossreactivities of the $\mathrm{PGE}_{2}, 6-\mathrm{keto}_{\mathrm{PGF}}, \mathrm{TxB}_{2}$, and $\mathrm{LTC}_{4}$ antisera have previously been described (9).

Microsphere flow mapping. Dry carbonized microspheres $16.0 \pm 0.3$ $\mu \mathrm{m}$ diam labeled either with ${ }^{51} \mathrm{Cr}$ or ${ }^{57} \mathrm{Co}$ were suspended in $84 \%$ dextrose solution containing several drops of $0.05 \%$ Tween 80 , dispersed by ultrasonication and mixed on a vortex shaker for $3 \mathrm{~min}$ before administration. To perform microsphere flow studies, normal or infarcted rabbit hearts were retrogradely perfused with Krebs-Henseleit buffer as described above. A 30-min equilibration was allowed for coronary perfusion pressure to stabilize. At this time $\sim 50,000$ microspheres $(0.1-\mathrm{ml}$ bolus injection) were injected into the perfusion apparatus $6 \mathrm{~cm}$ proximal to the aortic cannula. The number of microspheres injected was determined by gamma counting (Tracor 1191 gamma counter, Tracor, Inc., Austin, TX) the syringe and needle used for administration both before and after injection. $20 \mathrm{~min}$ after the first microsphere injection, a second radiolabeled microsphere injection was performed. This injection was made either under control conditions so as to validate the constancy of tissue flow, or after intracoronary bolus administration of $300 \mathrm{ng}$ of IMLP. Microsphere injections were made at the peak of the vasoconstriction (as monitored by coronary perfusion pressure) elicited by fMLP. In control hearts that did not show a vasoconstriction, microspheres were injected 3 min after fMLP administration, the time at which fMLP caused its peak vasoconstrictor effect. $10 \mathrm{~min}$ after the second microsphere injection, the hearts were perfused with $1 \%$ triphenyl tetrazolium chloride to delineate the infarct zone. The right and left atria subsequently were removed from the hearts and blotted with gauze. The ventricles were sliced along the posterior interventricular septum and divided into 100 200 -mg pieces that were blotted dry. The location of each of these pieces and their relation to the infarct zone were plotted on a map of the ventricles. The samples of atria and ventricle were placed in preweighed plastic vials, weighed, and counted on a gamma counter. A known number of ${ }^{51} \mathrm{Cr}$ and ${ }^{57} \mathrm{Co}$ microspheres were simultaneously counted to determine the counts per minute of a single microsphere. A computer (VAX 11/780) was used to correct the counts for interaction between the two isotopes as well as to calculate tissue flow (milliliters per minute per gram) and regional vascular resistance.

Radiochemistry experiments. Hearts were removed from anesthetized, heparinized rabbits, rinsed in ice-cold saline and perfused with $200 \mathrm{ml}$ of ice-cold saline to remove blood from the vasculature. The right and left atria, normal zones of right and left ventricle and infarcted zone of left ventricle were separated, blotted dry, weighed, and scissor minced. The tissues were placed in ice-cold $50 \mathrm{mM}$ Tris buffer $\mathrm{pH} 7.8$ containing $1 \mathrm{mM}$ EGTA at a concentration of $1 \mathrm{~g}$ of tissue per $3 \mathrm{ml}$ of buffer. The samples were homogenized in a homogenizer (Tekmar Co., Cincinnati, $\mathrm{OH}$ ) and centrifuged at $10,000 \mathrm{~g}$ for $15 \mathrm{~min}$ at $4^{\circ} \mathrm{C}$. The pellet was discarded and the resulting supernatant was used for incubations. 300 $\mu \mathrm{l}$ aliquots of the $10,000 \mathrm{~g}$ supernatant were incubated in $50 \mathrm{mM}$ Tris buffer pH 7.8 containing $5 \mathrm{mM} \mathrm{Ca}^{2+}, 1.2 \mathrm{mM}$ epinephrine, and $30 \mu \mathrm{M}$ $\left[{ }^{14} \mathrm{C}\right]$ arachidonate in a total volume of $0.5 \mathrm{ml}$. Reactions were run for $30 \mathrm{~min}$ at $37^{\circ} \mathrm{C}$ and terminated by acidification with $4 \mathrm{~N}$ formic acid. In inhibitor experiments the reaction mixture was preincubated for 5 min with BW-775C (final concentration $=173 \mu \mathrm{M}$ ) or indomethacin $(22 \mu \mathrm{M})$ and the reaction started by the addition of $\left[{ }^{14} \mathrm{C}\right]$ arachidonate. Products were extracted from the reaction mixture with 4 vol of ethyl acetate. The ethyl acetate was dried under $\mathrm{N}_{2}$ and the products were resuspended in $50 \mu \mathrm{l}$ of $\mathrm{CHCl}_{3} / \mathrm{MeOH}$ (2:1). The samples were then applied to a silica gel 60 plate and developed in a solvent system of $\mathrm{CHCl}_{3} / \mathrm{MeOH} /$ acetic acid/H $\mathrm{H}_{2} \mathrm{O}: 90: 8: 1: 0.8$. Authentic standards of $\mathrm{TxB}_{2}$, 6-keto prostacycline (PGF) $)_{1 \alpha}, \mathrm{PGE}_{2}, \mathrm{PGF}_{2 \alpha}, \mathrm{PGD}_{2}, \mathrm{PGA}_{2}, 12-\mathrm{Hete}$ and arachidonic acid were co-chromatographed with the samples. The plates were dried and autoradiograms produced by exposing the plates to film (Kodak X-Omat, Eastman Kodak Co., Rochester, NY) for 2 wk. The standards were visualized with iodine vapor and products were identified by comigration with the standards. 
Microsomal kinetics. To prepare microsomes, various regions of the heart were prepared as described above. The scissor-minced tissues were placed in $50 \mathrm{mM}$ Tris buffer $\mathrm{pH} 7.8$ containing $10 \mathrm{mM}$ EGTA and $1 \%$ bovine serum albumin ( $3 \mathrm{ml}$ buffer $/ \mathrm{g}$ of tissue). The tissues were homogenized in a Tekmar homogenizer and centrifuged at $10,000 \mathrm{~g}$ for $15 \mathrm{~min}$. The resultant supernatant was centrifuged at $100,000 \mathrm{~g}$ for $1 \mathrm{~h}$. The pellet from this centrifugation was resuspended in $50 \mathrm{mM}$ Tris buffer pH 7.8 containing $10 \mathrm{mM}$ EGTA $(0.25 \mathrm{ml}$ buffer $/ \mathrm{g}$ wet wt tissue) using a Dounce glass hand homogenizer. 4- $\mu \mathrm{l}$ aliquots of the microsomes were incubated in $50 \mathrm{mM}$ Tris buffer $\mathrm{pH} 7.8$ containing $1.2 \mathrm{mM}$ epinephrine (total volume $=100 \mu \mathrm{l}$ ) with unlabeled arachidonic acid ranging in concentration from 0.3 to $30 \mu \mathrm{M}$. The reactions were conducted at $37^{\circ} \mathrm{C}$ for $10 \mathrm{~min}$ and terminated by quick freezing in an acetone-dry ice bath. Preliminary experiments showed that the rate of microsomal prostaglandin synthesis was linear for at least $15 \mathrm{~min}$. For analysis the samples were thawed, debris was pelleted by centrifugation and the reaction mixture was assayed by specific RIA for $\mathrm{TxB}_{2}$ and $\mathrm{PGE}_{2}$. TxB $\mathrm{T}_{2}$ and $\mathrm{PGE}_{2}$ synthesis were normalized to the protein concentration of the microsomes as determined by the fluorescamine method (12). Apparent $K_{\mathrm{M}}$ and apparent $V_{\max }$ values were calculated using standard Lineweaver-Burk plots.

Histology. Hearts were removed from anesthetized heparinized rabbits and perfused through the vasculature with saline to remove blood and with $10 \%$ sodium phosphate-buffered formalin. The hearts were subsequently submerged in the formalin solution. Transmural slices of the heart were taken so as to include both the atria and the ventricle. Histologic sections were dehydrated in ethanol, embedded in paraffin, and $5-\mu \mathrm{m}$ sections were cut. Sections were stained with hematoxylin and eosin and examined with a light microscope. Sections of atria from normal and infarcted hearts were also stained with toluidine blue, aldehyde fuchsin, Alcian blue and a Ziehl-Neelson stain. These sections were examined by light microscopy for the presence or absence of mast cells.

\section{Results}

Agonist stimulation of isolated atria and ventricles. To determine which anatomic areas of the heart were responsible for agoniststimulated arachidonate metabolism, isolated atria and isolated perfused ventricles were prepared from normal rabbit hearts and from hearts that had sustained a left ventricular myocardial infarction $4 \mathrm{~d}$ previously. Each of these preparations was challenged with varying doses of either fMLP or BK. The eicosanoid synthetic responses elicited by IMLP at a dose of $100 \mathrm{ng}$ and BK at a dose of $1 \mu \mathrm{g}$ are presented in Table I. Under basal (no agonist) conditions the only metabolite produced in appreciable quantities by the normal heart was prostacyclin, and this product was synthesized largely in the ventricle. In the infarcted hearts, the cardiac atria but not ventricles produced significantly increased basal amounts of $\mathrm{PGE}_{2}$ and prostacyclin.

fMLP administration stimulated cardiac synthesis of $\mathrm{TxB}_{2}$, $\mathrm{PGE}_{2}$, and $\mathrm{LTC}_{4}$ in normal hearts. $\mathrm{LTC}_{4}$ synthesis was largely derived from ventricular tissue, whereas the synthesis of $\mathrm{TxB}_{2}$ and $\mathrm{PGE}_{2}$ occurred in both atria and ventricle. In the infarcted hearts $\mathrm{fMLP}$ caused an exaggerated synthesis of $\mathrm{TxB}_{2}, \mathrm{PGE}_{2}$, and prostacyclin. While the infarcted ventricle did produce four times more $\mathrm{TxB}_{2}$ than the normal ventricle, the increases in cardiac prostaglandin synthesis were largely attributable to enhanced atrial eicosanoid synthesis. Interestingly while fMLPstimulated peptidoleukotriene synthesis was not enhanced in the whole heart, there was a small but significant increase in the atrial synthesis of peptidoleukotrienes.

Bradykinin administration resulted in cardiac synthesis of $\mathrm{PGE}_{2}$ and prostacyclin in normal hearts. This synthesis occurred in both atria and ventricle roughly in proportion to the mass of the tissue (ventricular wet weight was roughly six times that of atria). In infarcted hearts $\mathrm{BK}$ stimulated $\mathrm{TxB}_{2}$ synthesis as well as grossly exaggerated synthesis of $\mathrm{PGE}_{2}$ and prostacyclin (Table I). While BK stimulated thromboxane synthesis in both the atria and the ventricles of infarcted hearts, enhanced $\mathrm{PGE}_{2}$ and prostacyclin synthesis was seen only in the cardiac atria. The sum of BK-stimulated atrial and ventricular $\mathrm{PGE}_{2}$ and prostacyclin synthesis does not nearly add up to the level of synthesis seen in the intact infarcted heart. This may reflect a major contribution from the atrial vasculature, since isolated atria are bathed

Table I. Agonist-stimulated Eicosanoid Synthesis in Isolated Atria and Ventricles from Normal and Infarcted Rabbit Hearts

\begin{tabular}{|c|c|c|c|c|c|c|c|c|}
\hline & \multicolumn{2}{|l|}{$\mathrm{T} \times \mathrm{BB}_{2}$} & \multicolumn{2}{|l|}{$\mathrm{PGE}_{2}$} & \multicolumn{2}{|c|}{ 6-KetoPGF $F_{1 \alpha}$} & \multicolumn{2}{|l|}{$\mathrm{LTC}_{4}$} \\
\hline & Normal & 4 D LVMI & Normal & 4 D LVMI & Normal & 4 D LVMI & Normal & 4 D LVMI \\
\hline & $n g / 5 \min$ & $n g / 5 \min$ & $n g / 5 \min$ & $n g / 5 \min$ & $n g / 5 \min$ & $n g / 5 \min$ & $n g / 5 \min$ & $n g / 5 \min$ \\
\hline \multicolumn{9}{|l|}{ Basal } \\
\hline Atria $(n=6)$ & $1 \pm 0.4$ & $3 \pm 1$ & $2 \pm 1$ & $33 \pm 8^{*}$ & $9 \pm 1$ & $33 \pm 6^{*}$ & $<1$ & $<1$ \\
\hline Ventricle $(n=6)$ & $<1.0$ & $3 \pm 1$ & $2 \pm 1$ & $2 \pm 1$ & $35 \pm 10$ & $40 \pm 11$ & $<1$ & $<1$ \\
\hline Intact heart $(n=7)$ & $<1.0$ & $6 \pm 4$ & $5 \pm 3$ & $37 \pm 13^{*}$ & $55 \pm 16$ & $84 \pm 15$ & $3 \pm 2$ & $3 \pm 1$ \\
\hline \multicolumn{9}{|l|}{ fMLP (100 ng) } \\
\hline Atria $(n=6)$ & $18 \pm 4$ & $133 \pm 33^{*}$ & $18 \pm 3$ & $108 \pm 29^{*}$ & $17 \pm 5$ & $89 \pm 17^{*}$ & $0.9 \pm 0.1$ & $2.3 \pm 0.2^{*}$ \\
\hline Ventricle $(n=6)$ & $9 \pm 4$ & $39 \pm 7^{*}$ & $24 \pm 9$ & $85 \pm 37$ & $71 \pm 28$ & $85 \pm 17$ & $30 \pm 7$ & $24 \pm 7$ \\
\hline Intact heart $(n=7)$ & $30 \pm 9$ & $235 \pm 66^{*}$ & $27 \pm 10$ & $330 \pm 108^{*}$ & $120 \pm 54$ & $714 \pm 285^{*}$ & $65 \pm 25$ & $89 \pm 22$ \\
\hline \multicolumn{9}{|l|}{ Bradykinin $(1 \mu \mathrm{g})$} \\
\hline Atria $(n=6)$ & $2 \pm 1$ & $8 \pm 3$ & $23 \pm 1$ & $134 \pm 25^{*}$ & $69 \pm 20$ & $207 \pm 59^{*}$ & $<1$ & $<1$ \\
\hline Ventricle $(n=6)$ & $3 \pm 1$ & $12 \pm 3^{*}$ & $244 \pm 57$ & $400 \pm 160$ & $404 \pm 56$ & $338 \pm 112$ & $<1$ & $<1$ \\
\hline Intact heart $(n=7)$ & $<1.0$ & $53 \pm 22^{*}$ & $221 \pm 15$ & $2512 \pm 313^{*}$ & $667 \pm 201$ & $5229 \pm 1125^{*}$ & $<1$ & $<1$ \\
\hline
\end{tabular}

Samples of coronary venous effluent or atrial bath buffer were collected for $5 \mathrm{~min}$ before agonist stimulation (basal), or immediately following challenge with either AMLP or BK. Collections were made from isolated atria in a tissue bath (atria), isolated perfused ventricles, or isolated perfused hearts (intact heart). These tissues were taken either from control rabbits (normal) or from rabbits that had sustained a surgical left ventricular myocardial infarction $4 \mathrm{~d}$ previously ( $4 \mathrm{~d} \mathrm{LVMI})$. All values are expressed as total nanograms released in $5 \mathrm{~min} \pm \mathrm{SEM}$. A two-tailed t-test for unpaired data was used to compare infarcted hearts with normal hearts. ${ }^{*} P<0.05$. 
in agonist whereas atria in intact hearts are challenged with agonist via the coronary vasculature. Alternatively, the discrepancy between BK-stimulated prostanoid synthesis in intact hearts and in isolated atria and ventricles may represent other idiosyncrasies of the preparations, such as the slower rate of contraction of isolated ventricles compared to intact hearts or possibly hypoxia of the isolated atria from infarcted hearts. It is also important to note that the data presented in Table I represent synthesis by whole atria or whole ventricle and are not corrected for mass. As the wet weight of the atria is only $15 \%$ that of the ventricles, atrial eicosanoid synthesis far exceeds that of ventricle on a per gram basis. Agonist-stimulated eicosanoid synthesis was dose dependent for $\mathrm{TxB}_{2}, \mathrm{PGE}_{2}$, and prostacyclin. This is illustrated for the fMLP-stimulated synthesis of thromboxane in Fig. 1.

The preceding data indicates that the cardiac atria are responsible for the majority of thromboxane synthesis in normal hearts and for the exaggerated agonist-stimulated thromboxane, $\mathrm{PGE}_{2}$ and prostacyclin synthesis observed in hearts with left ventricular myocardial infarction. To determine if agonist-stimulated prostaglandin synthesis was localized to the right or left cardiac atrium, agonist stimulations were performed on isolated right and left atria. In these studies only thromboxane and $\mathrm{PGE}_{2}$ synthesis were studied. The results of these experiments are shown in Table II. Under basal conditions $\mathrm{PGE}_{2}$ synthesis in the atria from infarcted hearts occurred predominantly in the right cardiac atrium. In the atria from normal and infarcted hearts, fMLP-stimulated thromboxane and $\mathrm{PGE}_{2}$ synthesis also occurred preferentially in the right cardiac atrium. Similarly, bradykinin-stimulated prostaglandin synthesis was predominantly localized to the right cardiac atrium (Table II).

Radiochemical enzyme localization. The dominant role of the right cardiac atrium in cardiac prostaglandin synthesis suggests localization of arachidonate-metabolizing enzymes to this chamber. To investigate this hypothesis, we prepared crude extracts $(10,000 \mathrm{~g}$ supernatant) from right and left cardiac atria, normal zones of ventricle and infarcted zones of ventricle. These extracts were incubated with $\left[{ }^{14} \mathrm{C}\right]$ arachidonate and the products were separated by thin-layer chromatography and visualized by autoradiography. The results of these studies are illustrated in Fig. 2. As can be seen, right cardiac atria from normal and infarcted hearts produce large amounts of eicosanoids that co-

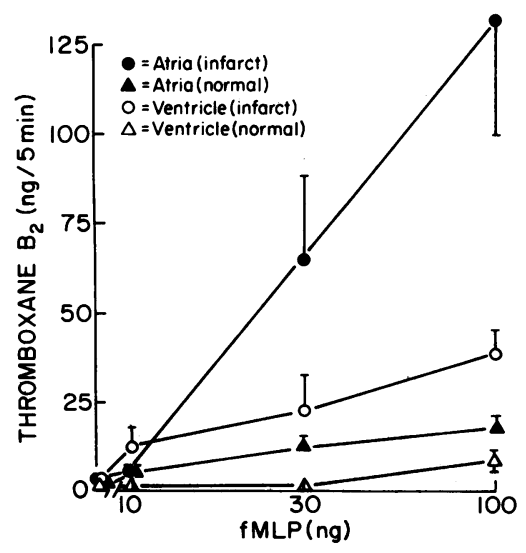

Figure 1. Dose-response curves for AMLP-stimulated thromboxane synthesis in atria and ventricles from normal and infarcted rabbit hearts. Isolated atria and isolated perfused ventricles were prepared from control rabbit hearts (normal) and from rabbit hearts subjected $4 \mathrm{~d}$ previously to left ventricular myocardial infarction (infarct), as described in Table I ( $n$

$=6$ ). Atria from normal hearts and infarcted hearts had respective wet weights of $0.55 \pm 0.06 \mathrm{~g}$ (mean $\pm \mathrm{SEM}$ ) and $0.66 \pm 0.03 \mathrm{~g}$; ventricles from normal and infarcted hearts had respective wet weights of $3.31 \pm 0.27 \mathrm{~g}$ and $3.74 \pm 0.57 \mathrm{~g}$.
Table II. Agonist-stimulated Prostaglandin Synthesis in Isolated Left and Right Atria from Normal and Infarcted Rabbit Hearts

\begin{tabular}{|c|c|c|c|c|}
\hline & \multicolumn{2}{|l|}{$\mathrm{TxB}_{2}$} & \multirow{2}{*}{\multicolumn{2}{|c|}{$\mathrm{PGE}_{2}$}} \\
\hline & \multirow[b]{2}{*}{ Normal } & \multirow{2}{*}{$\begin{array}{l}4 \text { D } \\
\text { LVMI }\end{array}$} & & \\
\hline & & & Normal & 4 D LVMI \\
\hline & $\begin{array}{l}\mathrm{ng} / 5 \\
\mathrm{~min}\end{array}$ & $\begin{array}{l}n g / 5 \\
\mathrm{~min}\end{array}$ & $n g / 5 \min$ & $\mathrm{ng} / 5 \mathrm{~min}$ \\
\hline \multicolumn{5}{|l|}{ Basal } \\
\hline Right atria $(n=6)$ & $1 \pm 0.4$ & $2 \pm 1$ & $1.1 \pm 0.9$ & $13 \pm 8$ \\
\hline Left atria $(n=6)$ & $<1.0$ & $1 \pm 0.7$ & $<1.0$ & $6 \pm 4$ \\
\hline \multicolumn{5}{|l|}{ fMLP (100 ng) } \\
\hline Right atria $(n=6)$ & $15 \pm 3^{*}$ & $36 \pm 7^{*}$ & $7 \pm 3$ & $53 \pm 19^{*}$ \\
\hline Left atria $(n=6)$ & $2 \pm 1$ & $5 \pm 1$ & $2 \pm 1$ & $9 \pm 4$ \\
\hline \multicolumn{5}{|l|}{ Bradykinin $(1 \mu \mathrm{g})$} \\
\hline Right atria $(n=6)$ & $4 \pm 2$ & $11 \pm 3^{*}$ & $23 \pm 8^{*}$ & $269 \pm 69^{*}$ \\
\hline Left atria $(n=6)$ & $1 \pm 0.2$ & $3 \pm 1$ & $3 \pm 1$ & $43 \pm 17$ \\
\hline
\end{tabular}

Atria were removed from control rabbit hearts (normal) and from rabbit hearts subjected $4 \mathrm{~d}$ previously to a left ventricular myocardial infarction (4 D LVMI). Right atria from normal and infarcted hearts had wet weights $( \pm$ SEM), respectively, of $0.26 \pm 0.04 \mathrm{~g}$ and $0.31 \pm 0.02$ $\mathrm{g}$; left atria from normal and infarcted hearts had respective wet weights of $0.29 \pm 0.03 \mathrm{~g}$ and $0.35 \pm 0.04 \mathrm{~g}$. A two-tailed $t$ test for unpaired data was used to compare right and left atria. ${ }^{*} P<0.05$.

migrate with $\mathrm{PGE}_{2}, \mathrm{PGF}_{2 \alpha}, \mathrm{TxB}_{2}$, and $\mathrm{PGD}_{2}$. At the top of the plate a band is seen in the zone where monohydroxyeicosatetraenoic acids (HETE) would be expected to migrate. In this solvent system $\mathrm{PGE}_{2}$ and 6-keto $\mathrm{PGF}_{1 \alpha}$ comigrate. Chromatograms developed in alternative solvent systems (data not shown) demonstrate that right atria produce similar amounts of $\mathrm{PGE}_{2}$ and 6-keto $\mathrm{PGF}_{1 \alpha}$. The conversion of arachidonic acid to the various products was qualitatively similar in atria from normal and infarcted hearts. The other chambers of the heart including left atrium, normal ventricle and infarcted zones of ventricle produced markedly smaller amounts of the products comigrating with $\mathrm{PGE}_{2}$ and $\mathrm{PGF}_{2 \alpha}$ than did right atria. Additionally, it is apparent that the synthesis of products comigrating with $\mathrm{TxB}_{2}$ and $\mathrm{PGD}_{2}$ is exclusively limited to the right cardiac atrium.

To validate the identification of the various arachidonate metabolites, incubations of the right atrial extract were performed in the presence of the cyclooxygenase inhibitor indomethacin and the combined cyclooxygenase-lipoxygenase inhibitor BW-

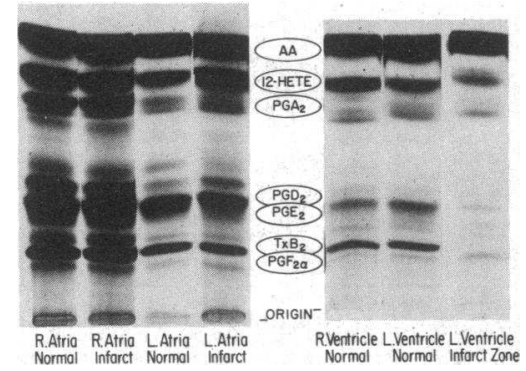

Figure 2. Radiochromatograms of eicosanoid synthesis by atria and ventricles from normal and infarcted (4 d) rabbit hearts. The percentage conversions of arachidonate in the various chambers of the heart were: normal right atria $47 \%$, right atria

from infarcted heart $35 \%$, normal left atria $17 \%$, left atria from infarcted heart $20 \%$, normal right ventricle $15 \%$, normal left ventricle $12 \%$, and infarcted left ventricle $9 \%$. 
755C (Fig. 3). Indomethacin inhibited synthesis of all products except for the band comigrating with 12-HETE. BW-755C inhibited the synthesis of both the product comigrating with monoHETEs, and the products comigrating with prostaglandin standards. The band comigrating with $\mathrm{PGA}_{2}$ was in part an autooxidation product of arachidonic acid, as it appeared as a faint band on chromatograms of arachidonic acid incubated without cell extract.

Kinetic analysis of thromboxane and $P G E_{2}$ synthesis. One possible mechanism whereby left ventricular myocardial infarction could enhance agonist-stimulated right atrial prostanoid synthesis would be by increasing the activity of arachidonate metabolizing enzymes. To test this hypothesis microsomes were prepared from the various chambers of either control hearts or hearts having sustained a left ventricular myocardial infarction $4 \mathrm{~d}$ previously. The microsomes were incubated with varying concentrations of arachidonic acid and the apparent $K_{\mathrm{m}}$ and apparent $V_{\max }$ were calculated for thromboxane and $\mathrm{PGE}_{2}$ synthesis. The results of these studies are presented in Table III. The $K_{\mathrm{m}}$ values for both thromboxane and $\mathrm{PGE}_{2}$ synthesis were similar in all chambers of both normal and infarcted hearts. The maximal rates of thromboxane and $\mathrm{PGE}_{2}$ synthesis were far greater in the right cardiac atrium than in any other zone of the heart. The maximal rates of thromboxane synthesis were virtually identical in right atria from normal and infarcted hearts. The

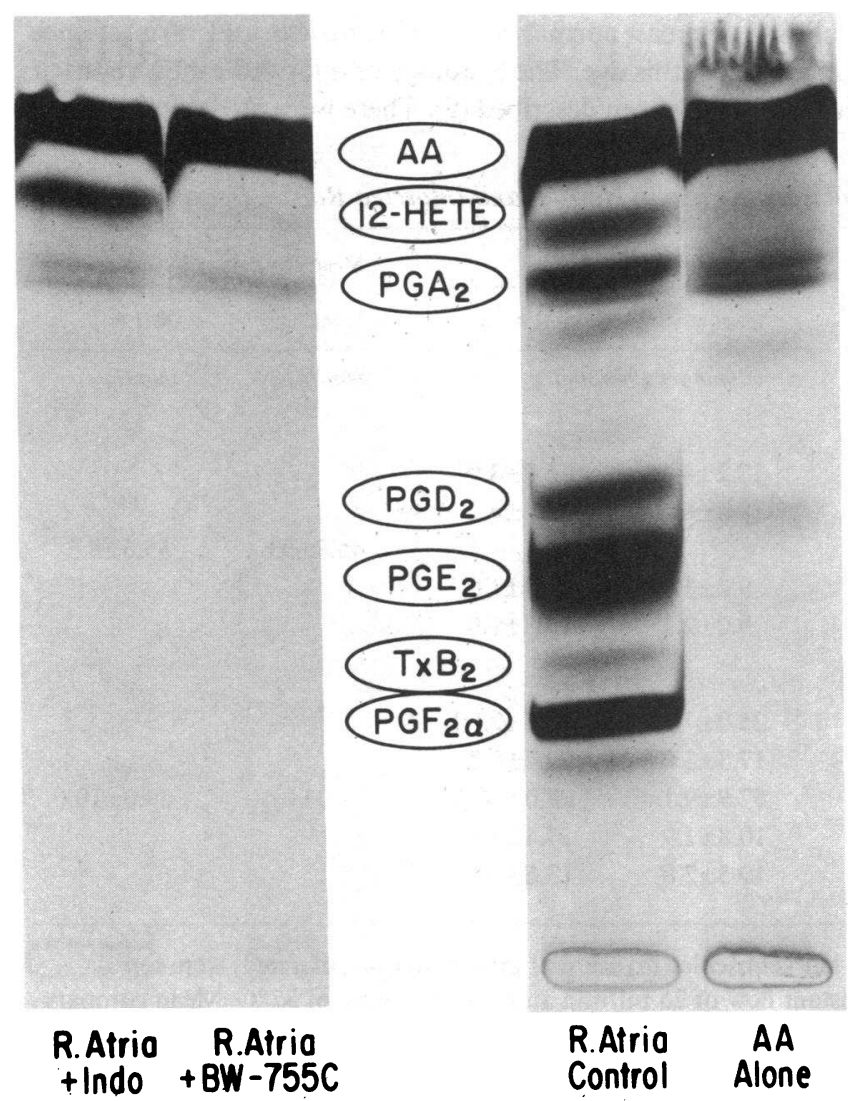

Figure 3. Radiochromatograms of right atrial eicosanoid synthesis in the presence and absence of specific enzyme inhibitors. The extracts were preincubated for $5 \mathrm{~min}$ with the cyclooxygenase inhibitor indomethacin (Indo; $22 \mu \mathrm{M}$ ) or the combined cyclooxygenase-lipoxygenase inhibitor BW-755C $(170 \mu \mathrm{M})$. The lane labeled "AA alone" represents a control incubation conducted in the absence of tissue extract. maximal rate of $\mathrm{PGE}_{2}$ synthesis was greater in normal right atria than in atria from hearts with left ventricular myocardial infarction. This data clearly demonstrates that the enhanced agonist-stimulated prostaglandin synthesis seen in atria from hearts with myocardial infarction is not the result of enhanced arachidonate metabolic capacity. It should be noted that as cyclooxygenase is the rate limiting step in the conversion of arachidonic acid to prostaglandins, the kinetic parameters determined largely reflect the activity of cyclooxygenase in the various tissues.

Microsphere flow studies. Microsphere flow mapping studies were performed to determine if fMLP-stimulated coronary vasoconstriction in infarcted rabbit hearts was localized to a specific region of the heart. Preliminary studies of microsphere injections in isolated perfused hearts showed that $>95 \%$ of the labeled microspheres were trapped in the heart, and that microsphere injection caused a minimal $(<5 \%)$ change in coronary perfusion pressure. Studies in which both ${ }^{51} \mathrm{Cr}$ and ${ }^{57} \mathrm{Co}$ microspheres were injected under control conditions showed that regional tissue flow was stable over time ( $20 \mathrm{~min})$ both in normal hearts and in hearts that had sustained a left ventricular myocardial infarction. Experiments were carried out by administering radiolabeled microspheres first under control conditions; 20 min later fMLP was administered, and at the peak of the coronary vasoconstriction ( $3 \mathrm{~min}$ ) microspheres labeled with a second radioisotope were administered. These experiments were performed on normal hearts and on hearts that had sustained a myocardial infarction $4 \mathrm{~d}$ previously, and in each case the animals served as their own control. Coronary perfusion pressure was monitored via a sidearm in the perfusion apparatus. Baseline perfusion pressure was the same in normal and infarcted hearts (Table IV).

The results of these studies are presented in Table IV. The wet weight of the cardiac atria, particularly the right atrium, was significantly greater in the hearts with ventricular infarction than in control hearts. No such difference was seen in the wet weights of ventricular tissue. The basal tissue flow (milliliters per minute per gram) and vascular resistance (milliliters per minute per gram per millimeter of mercury) values were similar in various regions of normal and infarcted hearts. The tissue flow was significantly reduced and vascular resistance significantly increased (as compared to normal left ventricle) in ventricular tissue from the infarct zone. In control hearts, administration of AMLP caused no significant change in either regional tissue flow or regional vascular resistance. This is consistent with the fact that fMLP did not cause a significant change in coronary perfusion pressure in normal rabbit hearts. When AMLP was administered to hearts with left ventricular myocardial infarction, tissue flow was significantly decreased in the right cardiac atria and there was a tendency toward increased vascular resistance in the right atria $(P<0.06$, normal vs. infarcted heart). No significant change was seen in tissue flow or vascular resistance in any other region of the heart following fMLP administration. IMLP did consistently increase coronary perfusion pressure in the infarcted hearts. The magnitude of the AMLP-induced vasoconstriction was variable, ranging from an 11 to $54 \%$ increase in perfusion pressure. When the percent increase in coronary perfusion pressure (over baseline) induced by $\mathrm{MLLP}$ in each of the infarcted heart preparations was plotted against the percent change in right atrial vascular resistance, a significant correlation was found $(r=0.97 ; P<0.05)$.

Gross and histologic analysis. Gross examinations of hearts with left ventricular myocardial infarction showed a blanched 
Table III. Kinetic Analysis of Thromboxane and PGE Synthetic Pathways in the Atria and Ventricles of Rabbit Heart

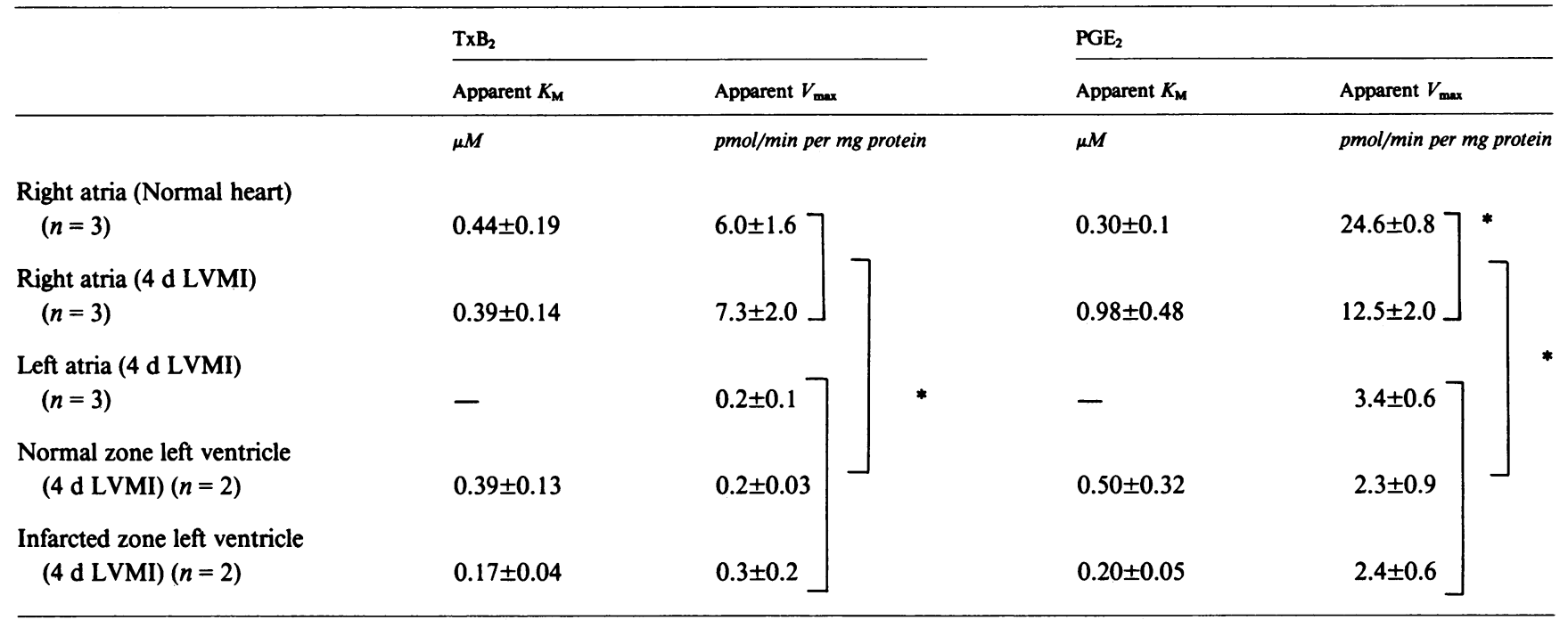

Right atria, left atria, normal zones of left ventricle and infarcted zones of left ventricle were removed either from normal rabbit hearts or from rabbit hearts subjected $4 \mathrm{~d}$ previously to a left ventricular myocardial infarction ( $4 \mathrm{~d}$ LVMI). Apparent $K_{\mathrm{M}}$ and apparent $V_{\max }$ values were determined from standard Lineweaver-Burk plots. Values are expressed either as mean $\pm \mathrm{SEM}$, or where $n=2$, as mean \pm range. Apparent $V_{\max }$ values for the various chambers of the heart were compared separately for $\mathrm{TxB}_{2}$ and $\mathrm{PGE}_{2}$ using a one-way analysis of variance followed by Duncan's multiple comparisons of the means. ${ }^{*} P<0.05$. No significant differences were found when apparent $K_{\mathrm{M}}$ values for the various chambers of the heart were compared either within $\left(\mathrm{TxB}_{2}\right.$ and $\mathrm{PGE}_{2}$ separately) or between ( $\mathrm{TxB}_{2}$ and $\mathrm{PGE}_{2}$ pooled) groups using an analysis of variance followed by Duncan's multiple comparison of the means.

area with intramyocardial hemorrhage at the apex of the heart. The infarcted area comprised 20 to $30 \%$ of the left ventricle on a wet weight basis. The infarcted area did not stain with tetra- zolium, whereas normal areas of ventricle and atria stained brightly with this dye. The histology of infarcted rabbit ventricle has previously been described (9). There were no detectable gross

Table IV. Microsphere Determinations of the Effects of fMLP on Regional Tissue Flow in Normal and Infarcted Rabbit Hearts

\begin{tabular}{|c|c|c|c|c|c|c|c|}
\hline & \multirow[b]{2}{*}{ Wet Weight } & \multicolumn{2}{|l|}{ Tissue flow } & \multicolumn{2}{|c|}{ Vascular resistance } & \multicolumn{2}{|c|}{ Mean coronary perfusion pressure } \\
\hline & & Basal & fMLP & Basal & fMLP & Basal & fMLP \\
\hline & $g$ & $\mathrm{ml} / \mathrm{min}$ per $\mathrm{g}$ & $\mathrm{ml} / \mathrm{min}$ per $\mathrm{g}$ & \multicolumn{2}{|c|}{$\mathrm{ml} / \mathrm{min}$ per $\mathrm{g}$ per $\mathrm{mmHg}$} & $m m H g$ & $m m H g$ \\
\hline \multicolumn{8}{|l|}{ Control $(n=4)$} \\
\hline Right atria & $0.27 \pm 0.02$ & $2.91 \pm 0.20$ & $3.66 \pm 0.41$ & $17.2 \pm 4.4$ & $15.9 \pm 3.0$ & & \\
\hline \multirow[t]{2}{*}{ Left atria } & $0.31 \pm 0.02$ & $2.86 \pm 0.46$ & $3.32 \pm 0.62$ & $19.4 \pm 6.6$ & $19.7 \pm 6.2$ & & \\
\hline & & & & & & $46.8 \pm 9.6$ & $55.6 \pm 8.3$ \\
\hline Left ventricle & $2.41 \pm 0.14$ & $5.34 \pm 0.13$ & $5.27 \pm 0.24$ & $9.2 \pm 1.4$ & $11.3 \pm 1.6$ & & \\
\hline Right ventricle & $0.95 \pm 0.13$ & $5.64 \pm 0.62$ & $5.48 \pm 0.32$ & $9.0 \pm 2.1$ & $10.3 \pm 1.6$ & & \\
\hline \multicolumn{8}{|c|}{ 4-d infarcted $(n=4)$} \\
\hline Right atria & $0.56 \pm 0.11^{*}$ & $2.35 \pm 0.37$ & $1.55 \pm 0.61^{*}$ & $25.0 \pm 7.3$ & $60.8 \pm 23.0$ & & \\
\hline Left atria & $0.46 \pm 0.03^{*}$ & $3.11 \pm 0.48$ & $3.32 \pm 0.58$ & $17.3 \pm 3.0$ & $22.7 \pm 5.8$ & & \\
\hline Infarct zone & $0.75 \pm 0.08$ & $1.43 \pm 0.30$ & $1.55 \pm 0.29$ & $52.9 \pm 9.1$ & $57.0 \pm 10.5$ & $51.0 \pm 6.4$ & $69.0 \pm 10.0$ \\
\hline Left ventricle & $1.89 \pm 0.38$ & $5.25 \pm 0.43$ & $5.60 \pm 0.51$ & $10.8 \pm 1.9$ & $13.4 \pm 2.9$ & & \\
\hline Right ventricle & $1.10 \pm 0.11$ & $5.71 \pm 0.98$ & $5.72 \pm 0.97$ & $10.5 \pm 2.8$ & $13.6 \pm 3.8$ & & \\
\hline
\end{tabular}

Normal (control) rabbit hearts and rabbit hearts subjected $4 \mathrm{~d}$ previously to a left ventricular myocardial infarction (4-d infarcted) were retrogradely perfused (Langendorff technique) with Krebs-Henseleit buffer at a constant flow of $25 \mathrm{ml} / \mathrm{min}$ and a temperature of $37^{\circ} \mathrm{C}$. Mean coronary perfusion pressure was continuously monitored via a sidearm in the perfusion apparatus. Microspheres $\left(16 \pm 0.3 \mu \mathrm{m} ;\right.$ labeled either with ${ }^{51} \mathrm{Cr}$ or ${ }^{57} \mathrm{Co}$ ) were injected into the perfusion apparatus either under basal conditions or following intracoronary bolus administration of $\mathrm{fMLP}$ ( $300 \mathrm{ng}$ ). Each preparation was studied under basal conditions and following AMP administration and therefore served as its own control. Following microsphere administration the hearts were stained with triphenyl tetrazolium chloride to delineate the infarct zone, and cut into 100-200-mg pieces. The location of each of these pieces was plotted on a map of the heart. The tissue samples were weighed and counted on a gamma counter. A VAX 11/780 computer was used to correct for the interaction between the two isotopes as well as to calculate tissue flow and regional vascular resistance. All values are expressed as the mean \pm SEM. A two-tailed $t$ test for unpaired data was used to compare the tissue wet weights from normal and infarcted hearts. $* P<0.05$. A two-tailed $t$ test for paired data was used to compare tissue flow and vascular resistance values in normal and infarcted hearts. 
anatomic differences between the atria from normal hearts and atria from hearts with myocardial infarction, and tetrazolium staining revealed no areas of necrosis in either preparation. Microscopic examinations of cardiac atria from normal and infarcted hearts were indistinguishable (i.e., no inflammatory cells were seen in either preparation), and in all atria apparently normal myocytes were loosely arranged around intact blood vessels and scattered interstitial fibroblasts. Staining of atrial sections with mast cell-specific stains failed to reveal the presence of significant numbers of mast cells in the atria from either normal or infarcted hearts. The vasculature of the atria from normal and infarcted hearts was also examined by light microscopy and no evidence of platelet aggregation or platelet-fibrin thrombi was found.

\section{Discussion}

These studies demonstrate several novel aspects of cardiac arachidonate metabolism in myocardial infarction. First, cyclooxygenase and thromboxane synthase in the heart are primarily localized in the right cardiac atrium. Second, the enhanced cardiac eicosanoid synthesis seen in rabbit ventricular myocardial infarction occurs predominantly in the right cardiac atrium, a site distant from the zone of injury. Finally, enhanced atrial eicosanoid synthesis in myocardial infarction is not mediated through an increase in the activity of arachidonate metabolizing enzymes.

In the normal rabbit heart, cyclooxygenase and thromboxane synthase are primarily localized in the right cardiac atrium. Synthesis of $\mathrm{PGE}_{2}, \mathrm{PGD}_{2}$, and $\mathrm{PGF}_{2 \alpha}$ also appear to be greatest in the right atria. It should, however, be noted that since $\mathrm{PGH}_{2}$ can be nonenzymatically transformed to $\mathrm{PGD}_{2}, \mathrm{PGE}_{2}$, and $\mathrm{PGF}_{2 \alpha}$ the data does not address the anatomic localization of the isomerases and reductases involved in the synthesis of these products. Consistent with this enzymatic localization, fMLPstimulated thromboxane synthesis occurs primarily in the atria. In contrast, fMLP-stimulated prostacyclin and peptidoleukotriene synthesis and bradykinin-stimulated $\mathrm{PGE}_{2}$ and prostacyclin synthesis occur primarily in the normal ventricle. These data indicate that despite the paucity of enzymatic activity in the ventricle, there are ventricular cell population(s) that are stimulated by fMLP with resultant synthesis of prostacyclin and leukotrienes, or recognize bradykinin with resultant synthesis of prostacyclin and $\mathrm{PGE}_{2}$. The identity of the ventricular cell type that synthesizes $\mathrm{PGE}_{2}$ and prostacyclin is indicated by previous investigations that have showed that coronary microvascular endothelia have the enzymatic capacity to synthesize these two prostanoids (13). Furthermore, studies in which the coronary vasculature of isolated perfused rabbit hearts is labeled with $\left[{ }^{14} \mathrm{C}\right]$ arachidonate indicate that bradykinin stimulates the synthesis of prostaglandins derived from this pool (14). While the cellular source of peptidoleukotrienes in the ventricle is unknown, we have previously hypothesized that mast cells located in the vascular adventitia may both bind IMLP and synthesize leukotrienes (9).

It is clear from this work that the cardiac atria contain cell populations with high enzymatic capacity for prostaglandin synthesis, and that these cells can respond to both FMLP and BK. It is unlikely that this agonist recognition occurs in resident inflammatory cells, as no macrophages or polymorphonuclear leukocytes were histologically detected in the atria. The ability of the atria to synthesize $\mathrm{PGD}_{2}$ and peptidoleukotrienes suggests that mast cells $(15,16)$ could be responsible for agonist-stimulated eicosanoid synthesis. However, efforts to histologically identify mast cells in the rabbit right atria using mast cell specific stains have been unsuccessful. It thus appears likely that a normal atrial cellular constituent (e.g., myocytes, fibroblasts, mesenchymal cells, endothelial cells) is responsible for both agonist binding and resultant eicosanoid synthesis. This is of particular interest, as functional fMLP receptors have previously been identified only on inflammatory cells $(17,18)$.

Enhanced cardiac arachidonate metabolism in myocardial infarction has previously been attributed to the inflammatory cells that infiltrate injured tissue and are known to prodigiously synthesize arachidonate metabolites $(19,20)$. Consistent with this hypothesis, we did observe enhanced agonist-stimulated thromboxane synthesis in the ventricles from infarcted hearts. It is surprising that the cardiac atria from hearts with left ventricular myocardial infarction exhibited exaggerated agoniststimulated synthesis of thromboxane, $\mathrm{PGE}_{2}$, prostacyclin, and peptidoleukotrienes. This enhanced atrial response to agonists was not associated with infiltration of the atria by inflammatory cells. The predominance of atrial eicosanoid synthesis in infarcted hearts is even more striking if product synthesis is corrected for the mass of the tissue. The atria from infarcted hearts produced more thromboxane, $\mathrm{PGE}_{2}$ and prostacyclin than the ventricle despite the fact that atrial mass (wet weight) is only $15 \%$ that of the ventricle.

Kinetic analyses of the enzymes involved in thromboxane and $\mathrm{PGE}_{2}$ synthesis indicate that exaggerated eicosanoid synthesis in atria from infarcted hearts is not the result of increased atrial prostanoid synthetic capacity. This suggests that exaggerated atrial eicosanoid synthesis results from increased availability of arachidonate substrate, resulting either from increased agonist (fMLP, BK) receptor recognition or from increased phospholipase activity in the atria from hearts with left ventricular injury. In addition to increased responsiveness to agonists, atria from hearts with ventricular infarction also had a greater wet weight than atria from control hearts. While this increased atrial mass was not nearly sufficient to account for the magnitude of enhanced atrial prostaglandin synthesis, it does suggest that left ventricular myocardial infarction results in atrial hypertrophy or hyperplasia.

The data from this study describe a phenomenon in which left ventricular injury results in enhanced right atrial response to BK and AMLP. The mechanism through which left ventricular injury influences the right atrium is unclear. It is possible that ventricular injury results in either a hemodynamic alteration (e.g., increased right atrial pressure) or a systemic hormonal effect to which the right atrium responds. Alternatively, soluble product(s) from the infarct zone may drain through the coronary venous bed to the right atrium and exert a stimulatory effect on agonist receptor expression or phospholipase activity.

The potential physiologic significance of the localization of eicosanoid synthetic enzymes to the right atrium is unknown, as is the potential pathophysiologic role of exaggerated right atrial eicosanoid synthesis in left ventricular myocardial infarction. The right atrium does play an important role in blood volume regulation, serving both as a sensory organ via its stretch receptors (21) and as an effector organ through secretion of atriopeptins (22). The localization of the sinoatrial node to the right atrium also makes this chamber uniquely important in the generation of the cardiac impulse. In light of these unique right atrial func- 
tions it will be of interest to determine if exaggerated atrial eicosanoid synthesis plays a role in either the abnormalities of volume regulation or the supraventricular dysrythmias seen after myocardial infarction. The data from microsphere flow studies do indicate one potential function for exaggerated atrial eicosanoid synthesis in myocardial infarction, namely regulation of atrial blood flow. The microsphere flow studies also provide an explanation for the fMLP-induced, leukotriene-mediated coronary vasoconstriction previously observed in infarcted rabbit hearts (9). In these studies fMLP-induced coronary vasoconstriction was localized to the right atrium, and was associated with enhanced atrial peptidoleukotriene synthesis.

In summary, we have demonstrated that the right cardiac atrium has far greater enzymatic capacity for eicosanoid synthesis than the other chambers of the rabbit heart. This synthetic capacity is unmasked in left ventricular myocardial infarction, and largely accounts for the enhanced agonist-stimulated eicosanoid synthesis seen in myocardial infarction. The potential pathophysiologic role of exaggerated eicosanoid synthesis in myocardial infarction, and the nature of the stimulus responsible for this phenomenon remain to be investigated.

\section{Acknowledgments}

This research was supported by National Institutes of Health Myocardial Ischemia Specialized Center of Research grant HL17646 to Dr. Needleman. Dr. Evers is a fellow of the Josiah Macy Foundation.

\section{References}

1. McCluskey, E. R., P. B. Corr, B. I. Lee, J. E. Saffitz, and P. Needleman. 1982. The arachidonic acid metabolic capacity of canine myocardium is increased during healing of acute myocardial infarction. Circ. Res. 51:743-750.

2. Mullane, K. M., N. Read, J. A. Salmon, and S. Moncada. 1984. Role of leukocytes in acute myocardial infarction in anesthetized dogs: relationship to myocardial salvage by anti-inflammatory drugs. J. Pharmacol. Exp. Ther. 228:510-522.

3. McCluskey, E. R., S. Murphree, J. E. Saffitz, A. R. Morrison, and P. Needleman. 1985. Temporal changes in 12-HETE formation in two models of canine myocardial infarction. Prostaglandins. 29:387.

4. Mullane, K. M., and S. Moncada. 1982. The salvage of ischaemic myocardium by BW-755C in anesthetized dogs. Prostaglandins. 24:255266.

5. Jolly, S. R., and B. R. Lucchesi. 1983. Effect of BW-755C in an occlusion-reperfusion model of ischemic myocardial injury. Am. Heart J. 106:8-13.

6. Bonow, R. O., L, C. Lipson, F. H. Sheehan, N. L. Capurro, J. M. Isner, W. C. Roberts, R. E. Goldstein, and S. E. Epstein. 1981. Lack of effect of aspirin on myocardial infarct size in the dog. Am. J. Cardiol. 47:258-264.
7. Jugdutt, B. I., G. M. Hutchins, B. J. Bulkley, B. Pitt, and L. C. Becker. 1979. Effect of indomethacin on collateral blood flow and infarct size in the conscious dog. Circulation. 59:734-743.

8. Bolli, R., R. E. Goldstein, N. Davenport, and S. E. Epstein. 1981. Influence of sulfinpyrazone and naproxen on infarct size in the dog. Am. J. Cardiol. 47:841-847.

9. Evers, A. S., S. Murphree, J. E. Saffitz, B. A. Jakschik, and P. Needleman. 1985. Effects of endogenously produced leukotrienes, thromboxane, and prostaglandins on coronary vascular resistance in rabbit myocardial infarction. J. Clin. Invest. 75:992-999.

10. Lefkowith, J. B., V. Flippo, H. Sprecher, and P. Needleman. 1985. Paradoxical conservation of cardiac and renal arachidonate content in essential fatty acid deficiency. J. Biol. Chem. 260:15736-15744.

11. Maclouf, J., M. Pradel, P. Pradelles, and F. Dray. $1976 .{ }^{125} \mathrm{I}$ derivatives of prostaglandins: a novel approach in prostaglandin analysis by radioimmunoassay. Biochim. Biophys. Acta. 431:139-146.

12. Udenfriend, S., S. Stein, P. Bohlen, W. Dairman, W. Leimgruber, and M. Weigele. 1972. Applications of fluorescamine, a new reagent for assay of amino acids, peptides, proteins and other primary amines in the picomole range. Science (Wash. DC). 178:871-872.

13. Gerritsen, M. E., and C. D. Cheli. 1983. Arachidonic acid and prostaglandin endoperoxide metabolism in isolated rabbit coronary microvessels and isolated and cultivated microvessel endothelial cells. $J$. Clin. Invest. 72:1658-1671.

14. Hsueh, W., and P. Needleman. 1978. Sites of lipase activation and prostaglandin synthesis in isolated perfused rabbit hearts and hydronephrotic kidneys. Prostaglandins. 16:661-681.

15. Roberts, L. J., R. A. Lewis, J. A. Oates, and K. F. Austen. 1979. Prostaglandin, thromboxane and 12-hydroxy-5,8,10,14 eicosatetraenoic acid production by ionophore-stimulated rat serosal mast cells. Biochim. Biophys. Acta. 575:185-192.

16. Razin, E., J. M. Mencia-Huerta, R. A. Lewis, E. J. Corey, and K. F. Austen. 1982. Generation of leukotriene $C_{4}$ from a subclass of mast cells differentiated in vitro from mouse bone marrow. Proc. Natl. Acad. Sci. USA. 79:4665-4667.

17. Aswanikumar, S., B. Corcoran, and E. Schiffmann. 1977. Demonstration of a receptor on rabbit neutrophils for chemotactic peptides. Biochem. Biophys. Res. Commun. 74:810-817.

18. Snyderman, R., and E. J. Fudman. 1980. Demonstration of a chemotactic factor receptor on macrophages. J. Immunol. 124:27542757.

19. Morley, J. M., A. Bray, R. W. Jones, D. H. Nugteren, and D. A. van Dorp. 1979. Prostaglandin and thromboxane production by human and guinea pig macrophages and leukocytes. Prostaglandins. 17:730736.

20. Bokoch, G. M., and P. W. Reed. 1980. Stimulation of arachidonic acid metabolism in the polymorphonuclear leukocyte by an $\mathrm{N}$-formylated peptide. J. Biol. Chem. 255:10223-10226.

21. Fater, D. C., H. D. Schultz, W. D. Sundet, J. S. Mapes, and K. L. Goetz. 1982. Effects of left atrial stretch in cardiac-denervated and intact conscious dogs. Am. J. Physiol. 242:H1056-H1064.

22. Manning, P. T., D. Schwartz, N. C. Katsube, S. W. Holmberg, and P. Needleman. 1985. Vasopressin-stimulated release of atriopeptin: endocrine antagonists in fluid homeostasis. Science (Wash. DC). 229: 395-397. 